\title{
Glagovian remodelling, plaque composition, and stenosis generation
}

Necropsy and intravascular ultrasound (IVUS) studies in vivo show that while the angiogram is good at detecting high grade stenosis it is very insensitive for demonstrating the actual extent of atherosclerosis. Segments of an angiographically normal coronary artery can harbour many occult plaques. ${ }^{1}$ There are two reasons for this insensitivity. The first is that behind the plaque itself the media may vanish allowing the plaque to bulge into the adventitia rather than toward the lumen. ${ }^{2}$ In extreme cases the internal elastic lamina breaks allowing the plaque to be extruded from the artery wall. The second process is that of arterial remodelling (compensatory dilatation) described by Glagov. ${ }^{3}$ In this process when a plaque develops the arterial media remodels to allow the vessel to increase its cross sectional area and thereby accommodate the plaque without any reduction in lumen area. At the site of a plaque the vessel cross sectional area can be increased by anything up to twice that found at an adjacent reference segment of normal artery. What is interesting, and not well understood, is that the degree of remodelling varies widely from plaque to plaque. Within one coronary artery there may be a plaque with no remodelling, while in another segment of the same artery at the site of a plaque there may be a $100 \%$ increase in the artery cross sectional area. ${ }^{1}$ Remodelling is plaque specific rather than patient specific.

\section{Generation of coronary stenosis}

The way in which high grade chronic stenosis is generated cannot be regarded solely as a function of plaque growth. Stenosis develops when the balance between plaque growth and the remodelling response is altered. It is emerging that a failure of the remodelling response is a factor of equal importance to increasing plaque size in generating stenosis.

Remodelling can be studied only in necropsy material where the arteries have been perfused at systemic pressure or by IVUS in vivo. The former has the advantage that the whole coronary tree can be examined, but the patients studied are highly selected to those dying of their disease. The latter has the disadvantage that only a limited number of plaques can be studied in any one individual. Both can only provide a snapshot view of the process at one moment in time.

\section{Definition of remodelling}

The degree of remodelling (compensatory dilatation) is calculated by comparing the cross sectional area of the artery at the site of a culprit plaque with that of an adjacent reference segment of normal artery. Those using IVUS have adopted an index for grading the degree of remodelling. While this method allows direct comparison between different studies it is just one way of subdividing a continuous variable. The term compensatory dilatation or remod- elling is applied for an increase of more than $5 \%$ in the cross sectional area (index >1.05) and remodelling regarded as absent when there is an increase of less than $5 \%$ (index < 1.05). Negative remodelling is a term used when the vessel cross sectional area is much less than that seen in an adjacent normal reference segment. ${ }^{4}$ This term introduces the concept that fibrous constriction may be another factor in general stenosis.

It is also possible to define remodelling as adequate or inadequate in a different way based on whether stenosis is present. If stenosis is present remodelling must be inadequate. The problem with such an approach is that an increase in overall cross sectional area of the artery of $75 \%$, for example, can be associated with stenosis when the plaque size is unusually large. Yet another approach is simply to use the per cent remodelling, positive or negative, as a continual variable to be treated as such for correlation with other parameters.

\section{Plaque disruption}

The study in this issue of Heart by von Birgelen and colleagues $^{5}$ casts some light on what plaque factors are associated with positive compensatory remodelling. It is now soundly established that culprit plaques which undergo an acute episode of disruption have certain key features. These include a large lipid core, a high macrophage content, a low smooth muscle content, and a thin cap. The lipid core is a space in the collagenous tissue of the intima containing cholesterol rich lipid debris. Core size can be determined by IVUS, particularly if the lipid within the core has been displaced by the entry of blood

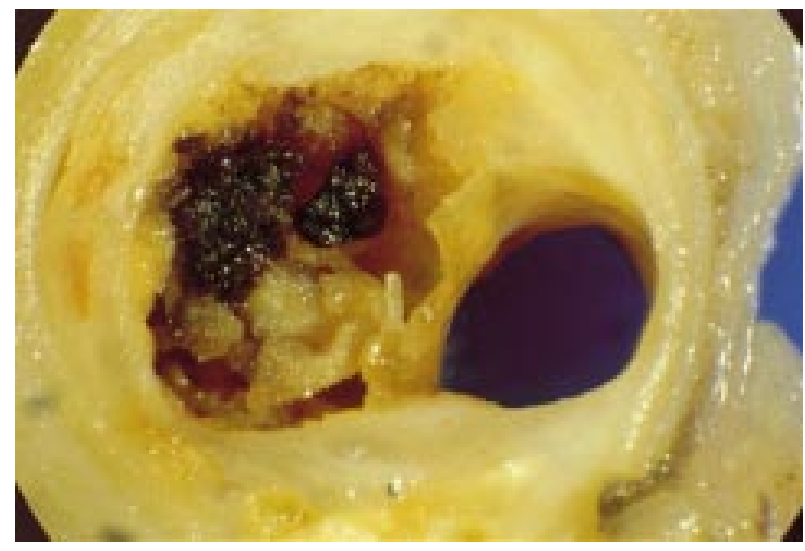

Figure 1 A plaque five days after thrombolysis. There is a cavity containing some residual thrombus which communicates with the arterial lumen via a hole in the plaque cap. The lipid in the core has been washed out in vivo to create the cavity. Reproduced from Davies MF. Atlas of coronary artery disease, with permission of the publishers

Lippincott-Raven. 


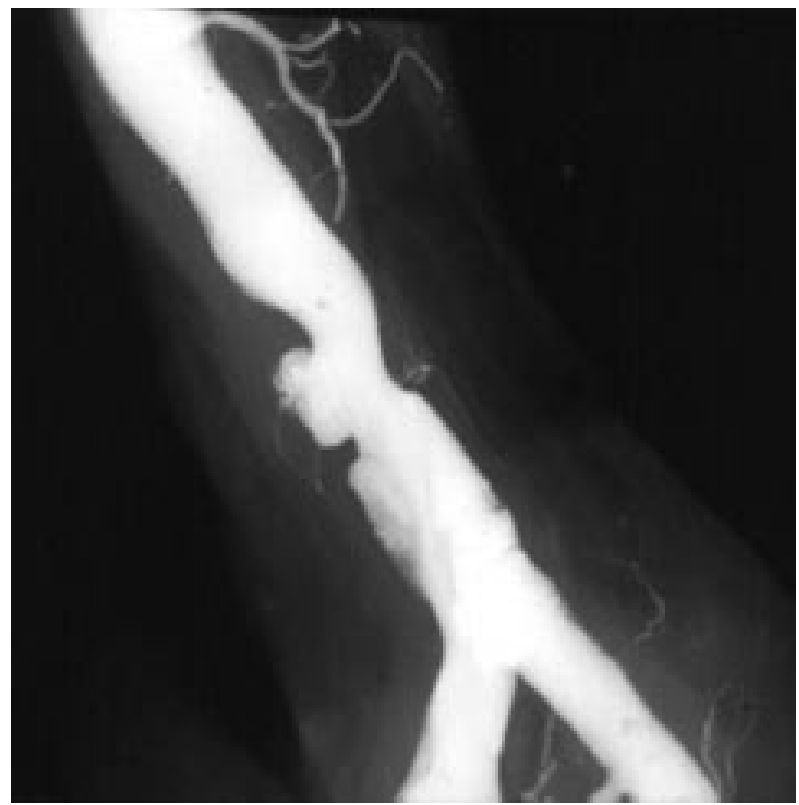

Figure 2 Postmortem angiogram showing a central cavity in a plaque the edges of which can still be seen. Reproduced from Davies MF. Atlas of coronary artery disease, with permission of the publishers Lippincott-Raven.

from the lumen via a tear in the cap. The core cavity is particularly striking after lysis has removed thrombus (figs 1 and 2).

\section{Mechanisms of remodelling}

The study reported in this issue ${ }^{5}$ shows that core size as defined by IVUS of culprit lesions for acute coronary syndromes is positively associated with previous positive remodelling (compensatory dilatation) of the artery at the site of the plaque. Of the 51 lesions studied 36 had compensatory enlargement (71\%) while 15 (29\%) did not. The core size was significantly larger in the group where compensatory enlargement had occurred. These findings have the important implication that many vulnerable plaques at risk of future acute thrombotic events will have undergone sufficient remodelling to be angiographically invisible. This fits well with the angiographic data showing that the thrombi which cause infarction often do not develop at sites of previous high grade stenosis ${ }^{6}$. More important, however, is the suggestion that core size and positive remodelling are linked. The link may be causal. Circumferential wall stress across the plaque with a large lipid core is altered. The core, being soft and deformable, cannot carry this stress which has to be redistributed to adjacent areas of the vessel wall.

Previous work on stress distribution using finite element analysis concentrated on the increased forces exerted on the plaque cap, ${ }^{7}$ but behind the plaque force is also increased which may potentiate stretching of the tissue and therefore remodelling. The association of a large lipid core and positive remodelling may be indirect. Remodelling of the arterial media depends on the complex interaction of growth factors and metalloproteinases concerned both with smooth muscle cell movement and degradation of connective tissue matrix. Large lipid cores are associated with high macrophage densities and these cells are major stimulators and producers of metalloproteinases. It may therefore be that core size is simply a surrogate for plaques with high macrophage activity, and it is this factor which enhances remodelling.

St George's Hospital Medical School,

M J DAVIES

BHF Cardiovascular Pathology,

Cranmer Terrace,

London SW17 ORE, UK

1 Varnava A. Coronary artery remodelling. Heart 1998;79:109-10.

2 Crawford T, Levene C. Medial thinning in atheroma. 7 Pathol Bacteriol 1953;66:19-23.

3 Glagov S, Weisenberd E, Zarins C, et al. Compensatory enlargement of human atherosclerotic coronary arteries. $N$ Engl f Med 1987;316:1371-5.

4 Pasterkamp G, Wensing P, Post M, et al. Paradoxical arterial wall shrinkage may contribute to luminal narrowing of human atherosclerotic femoral arteries. Circulation 1995;91:1444-9.

5 von Birgelen C, Klinkhart W, Mintz GS, et al. Size of emptied plaque cavity following spontaneous rupture is related to coronary dimensions, not to the degree of lumen narrowing. A study with intravascular ultrasound in vivo. Heart 2000;84:483-8.

6 Falk E, Shah P, Fuster V. Coronary plaque disruption. Circulation 1995;92: 656-71.

7 Richardson P, Davies M, Born G. Influence of plaque configuration and stress distribution on fissuring of coronary atherosclerotic plaques. Lancet 1989;ii:941-4.

\section{STAMPS IN CARDIOLOGY}

\section{Institutions}

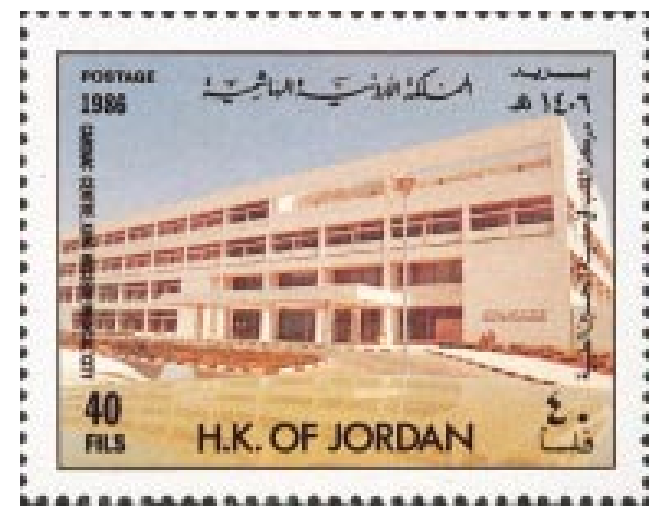

In 1986 Jordan issued three stamps commemorating the King Hussein Medical City. The 60 fils and 100 fils stamps concerned surgery and operating theatres. The 40 fils shows the Cardiac Centre building. 